\title{
Compassionate use of bedaquiline in highly drug-resistant tuberculosis patients in Mumbai, India
}

\author{
To the Editor:
}

Bedaquiline, a mycobacterial ATP synthase inhibitor [1], is the first new tuberculosis (TB) drug in almost 50 years. So far, over 800 patients have received the drug globally through compassionate use (CU) programmes. Drug-resistant TB has emerged as global public health priority [2] and TB strains are being reported with increasing levels of resistance, such as those that are multidrug resistant (MDR), extensively drug resistant (XDR) and beyond [2-4]. India bears the burden of the largest number of MDR cases in the world [2] and alarming patterns of resistance are frequently encountered in urban hot-spots like Mumbai, including the first Indian cases resistant to all first-line drugs and second-line drugs (SLDs) [5]. Several studies around the world have shown increased treatment efficacy after addition of bedaquiline to optimised background regimens (OBRs) [6-8]. In such settings, newer drugs like bedaquiline are urgently needed; however, accessing them has proven to be challenging in India. Of the 76 applications received from India, no more than 52 Indian patients throughout the country have received bedaquiline under the CU programme (personal communication: Janssen Pharmaceuticals, India, August 2, 2016). At the P.D. Hinduja hospital, a large private hospital in Mumbai, we have been treating patients with MDR-TB for the last two decades [9]. Between January 2013 and September 2015, 27 patients with drug-resistant TB strains were considered for bedaquiline. Applications for all 27 patients were made to the Janssen pharmaceuticals CU programme and, of these, 20 were approved and bedaquiline was administered. The resulting cohort is the largest in India to have received bedaquiline (20 patients from a countrywide total of 52). Bedaquiline was considered for patients based on the clinical judgement of the treating physician and there was no consultation with any panel of experts or TB consilium at this stage.

The mean time from application for bedaquiline to its availability in hand was 45-60 days. The reasons for bedaquiline being denied (seven patients) included incidence of TB-meningitis (two patients), age being less than 18 years (one patient) and less than three effective drugs in the background regimen (four patients). Bedaquiline was procured from Janssen Pharmaceuticals, Belgium and was provided free of charge on a CU basis. Only those patients not responding to their initial SLD regimen were considered for bedaquiline on CU grounds, access to which involved a laborious and fairly lengthy process (including an online application, review by the Janssen review board, application for the import permit in each individual case, dispatch, shipping and customs clearance before eventual delivery to our centre). All drugs in the regimen were administered in an ambulatory setting at home under the supervision of a designated family member or caregiver.

The results are outlined in table 1. The patients' age ranged from 18-40 years (mean 27.5 years). Of this cohort, 14 out of 20 patients were female, one patient was HIV positive (table entry 12) and one had diabetes mellitus (table entry 11). The mean body mass index (BMI) was $17.42 \mathrm{~kg} \cdot \mathrm{m}^{-2}$ and the majority of patients had advanced, cavitary (19 out of 20), bilateral (14 out of 20) disease with highly resistant patterns evident in drug susceptibility testing (DST) conducted prior to starting bedaquiline treatment (12 out of 20 had XDR-TB, while eight out of 20 had MDR-TB with additional fluoroquinolone resistance). This was a highly resistant group of patients with resistance to between five and 12 drugs (average 9.25 drugs). All 20 patients had received a mean of 6.1 SLDs for a period of between four and 126 months (mean 21.3 months) prior to being commenced on bedaquiline treatment. All 20 patients had pulmonary $\mathrm{TB}$, with two of the 20 having concomitant extrapulmonary TB (table entries 4 and 5).

@ERSpublications

Bedaquiline is a promising new drug treatment for drug-resistant TB. Urgent access is required in selected centres http://ow.ly/o1l73074ugh

Cite this article as: Udwadia ZF, Ganatra S, Mullerpattan JB. Compassionate use of bedaquiline in highly drug-resistant tuberculosis patients in Mumbai, India. Eur Respir J 2017; 49: 1601699 [https://doi.org/ $10.1183 / 13993003.01699-2016]$. 
TABLE 1 Profile and outcome for patients receiving bedaquiline under the compassionate use program at P.D. Hinduja Hospital and Medical Research Center,

Mumbai

\begin{tabular}{|c|c|c|c|c|c|c|c|c|c|c|c|c|}
\hline Entry & Sex & Age & Site & $\begin{array}{c}\text { Tests at } \\
\text { baseline }\end{array}$ & Resistance profile & $\begin{array}{l}\text { SLD Duration } \\
\text { months }\end{array}$ & $\begin{array}{l}\text { Lobes and } \\
\text { cavities }\end{array}$ & $\mathrm{OBR}^{+}$ & Surgery & $\begin{array}{l}\text { Weight } \\
\text { gain kg }\end{array}$ & $\begin{array}{l}\text { Time }^{\S} \\
\text { months }\end{array}$ & Outcome \\
\hline 1 & Male & 27 & Pul & $\mathrm{SS}+/ \mathrm{SC}+$ & $\begin{array}{c}\text { Pre-XDR (Fq): } \\
H, R, Z, E, \text { Sm, Ofx, } \\
\text { Mfx, Eto }\end{array}$ & 11 & $B / L(3) ; Y$ & Lzd, Cfz, PAS, Cs (4) & No & 6 & 1 & Cured \\
\hline 2 & Female & 28 & Pul & $\mathrm{SS}+/ \mathrm{SC}_{+}$ & $\begin{array}{c}\text { Pre-XDR (Fq): } \\
H, R, Z, E, S m, \text { Ofx } \\
\text { Mfx, Eto }\end{array}$ & 36 & $\mathrm{~B} / \mathrm{L}(5) ; \mathrm{Y}$ & $\begin{array}{c}\text { Lzd, Cfz, PAS, Cm, } \\
\text { Meropenem, Amox+Clav } \\
\text { (6) }\end{array}$ & No & 10 & 5 & $\begin{array}{l}\text { Relapsed (SC+ } 16 \text { months after } \\
\text { culture conversion) } \\
\text { Reapplying for bedaquiline and } \\
\text { also for delamanid } \\
\text { OBR ongoing }\end{array}$ \\
\hline 3 & Female & 40 & Pul & SS-/SC- & $\begin{array}{c}\text { XDR: } \\
\text { H, R, Z, E, Sm, } \\
\text { Ofx, Mfx, Eto, } \\
\text { Km, Am, Cm }\end{array}$ & 7 & $U / L(1) ; Y$ & $\begin{array}{c}\text { Lzd, Cfz, PAS, Cs, Cm, E } \\
(6)\end{array}$ & No & 7 & $\begin{array}{l}\text { Negative at } \\
\text { baseline }\end{array}$ & Cured \\
\hline 4 & Female & 29 & $\begin{array}{l}\text { Pul } \\
+ \text { Pl }\end{array}$ & SS-/SC- & $\begin{array}{l}\text { XDR: } \\
\mathrm{H}, \mathrm{R}, \mathrm{Z}, \mathrm{E}, \mathrm{Sm}, \\
\mathrm{Ofx}, \mathrm{Mfx}, \mathrm{Eto} \\
\mathrm{Km}, \mathrm{Am}, \mathrm{Cm}\end{array}$ & 11 & $\mathrm{~B} / \mathrm{L}(4) ; Y$ & $\begin{array}{l}\text { Lzd, Cfz, PAS, Clari, } \\
\text { Amox+Clav (5) }\end{array}$ & No & 1 & $\begin{array}{l}\text { Negative at } \\
\text { baseline }\end{array}$ & Cured \\
\hline 5 & Female & 20 & $\begin{array}{l}\text { Pul } \\
+ \text { Pl }\end{array}$ & $\mathrm{SS}+/ \mathrm{SC}+$ & $\begin{array}{c}\text { XDR: } \\
\text { H, R, Z, E, Sm, Ofx, } \\
\text { Mfx, Eto, PAS, Km }\end{array}$ & 16 & $\mathrm{~B} / \mathrm{L}(2) ; \mathrm{Y}$ & $\begin{array}{c}\text { Lzd, Cfz, PAS, Cs, Cm, } \\
\text { Eto }(6)\end{array}$ & No & 6 & - & $\begin{array}{c}\text { Nonresponder (SS-/SC+, at } \\
6 \text { months of bedaquiline) } \\
\text { Disease progressive } \\
\text { Received delamanid } \\
\text { sequentially } \\
\text { Currently SS+/SC+ } \\
\text { OBR ongoing }\end{array}$ \\
\hline 6 & Male & 25 & Pul & $\mathrm{SS}-/ \mathrm{SC}+$ & $\begin{array}{c}\text { XDR: } \\
H, R, Z, E, S m, O f x \\
\text { Mfx, Eto, PAS, Km, } \\
\text { Am, Cm }\end{array}$ & 2 & $U / L(2) ; Y$ & $\begin{array}{c}\text { Lzd, Cfz, PAS, Cs, Cm, } \\
\text { Mfx (6) }\end{array}$ & No & 6 & 2 & Cured \\
\hline 7 & Female & 23 & Pul & $\mathrm{SS}+/ \mathrm{SC}+$ & $\begin{array}{c}\text { XDR: } \\
\text { H, R, Z, E, Sm, } \\
\text { Ofx, Mfx, Eto, } \\
\mathrm{Km}, \mathrm{Am}, \mathrm{Cm}\end{array}$ & 43 & $U / L(2) ; Y$ & Lzd, Cfz, PAS, Cs, Thr (5) & No & 0 & - & $\begin{array}{c}\text { Nonresponder (SS+/SC+, at } \\
6 \text { months of bedaquiline) } \\
\text { Disease progressive } \\
\text { Currently SS+/SC+ } \\
\text { OBR ongoing }\end{array}$ \\
\hline 8 & Female & 31 & Pul & $\mathrm{SS}+/ \mathrm{SC}+$ & $\begin{array}{l}\text { Pre-XDR }(F q): \\
\text { H, R, Z, E, Sm, } \\
\text { Ofx, Mfx, Eto }\end{array}$ & 46 & $\mathrm{~B} / \mathrm{L}(5) ; \mathrm{Y}$ & $\begin{array}{c}\text { Lzd, Cfz, PAS, Cs, Cm, } \\
\text { Eto }(6)\end{array}$ & No & -1 & - & $\begin{array}{l}\text { Nonresponder (SS+/SC+, at } \\
6 \text { months of bedaquiline) } \\
\text { Disease progressive } \\
\text { Currently SS+/SC+ } \\
\text { OBR ongoing }\end{array}$ \\
\hline 9 & Female & 25 & Pul & $\mathrm{SS}+/ \mathrm{SC}+$ & $\begin{array}{c}\text { XDR: } \\
\text { H, R, Z, E, Sm, } \\
\text { Ofx, Mfx, Eto, } \\
\text { Km, Am, Cm }\end{array}$ & 6 & $B / L(3) ; Y$ & $\begin{array}{c}\text { Lzd, Cfz, PAS, Cs, Cm, } \\
\text { Eto, Cm, Meropenem, } \\
\text { Amox+Clav (9) }\end{array}$ & $\begin{array}{c}\text { Yes (left } \\
\text { pneumonectomy) }\end{array}$ & 1 & - & $\begin{array}{c}\text { Nonresponder (SS+/SC+, at } \\
6 \text { months of bedaquiline) } \\
\text { Disease progressive } \\
\text { Currently SS+/SC+ } \\
\text { OBR ongoing }\end{array}$ \\
\hline 10 & Female & 22 & Pul & $\mathrm{SS}+/ \mathrm{SC}+$ & $\begin{array}{l}\text { Pre-XDR }(F q): \\
\text { H, R, Z, E, Sm, } \\
\text { Ofx, Mfx, PAS }\end{array}$ & 7 & $\mathrm{~B} / \mathrm{L}(3) ; \mathrm{N}$ & Lzd, Cfz, Cs, Km, Eto (5) & No & 8 & 3 & Cured \\
\hline
\end{tabular}




\begin{tabular}{|c|c|c|c|c|c|c|c|c|c|c|c|c|}
\hline Entry & Sex & Age & Site & $\begin{array}{c}\text { Tests at } \\
\text { baseline }\end{array}$ & Resistance profile & $\begin{array}{l}\text { SLD Duration } \\
\text { months }\end{array}$ & $\begin{array}{l}\text { Lobes and } \\
\text { cavities }\end{array}$ & $\mathrm{OBR}^{+}$ & Surgery & $\begin{array}{l}\text { Weight } \\
\text { gain kg }\end{array}$ & $\begin{array}{l}\text { Time } \\
\text { months }\end{array}$ & Outcome \\
\hline 11 & Male & 29 & Pul & $\mathrm{SS}+/ \mathrm{SC}+$ & $\begin{array}{l}\text { Pre-XDR }(F q): \\
\text { H, R, Z, E, Sm, } \\
\text { Ofx, Mfx, Eto }\end{array}$ & 12 & $B / L(3) ; Y$ & $\begin{array}{c}\text { Lzd, Cfz, PAS, Cs, Cm, } \\
\text { Amox+Clav (6) }\end{array}$ & $\begin{array}{l}\text { Yes (bilateral upper } \\
\text { lobectomy) }\end{array}$ & 13 & 2 & Cured \\
\hline 12 & Male & 40 & Pul & $\mathrm{SS}+/ \mathrm{SC}+$ & $\begin{array}{c}\text { XDR: } \\
\text { H, R, Z, E, Sm, } \\
\text { Ofx, Mfx, Eto, } \\
\text { PAS, Km, Am }\end{array}$ & 12 & $\mathrm{~B} / \mathrm{L}(2) ; \mathrm{Y}$ & $\begin{array}{l}\text { Lzd, Cfz, Cs, Cm, Amox } \\
+ \text { Clav, Thr, Rifabutin (7) }\end{array}$ & $\begin{array}{c}\text { Yes (left } \\
\text { pneumonectomy) }\end{array}$ & 3 & 3 & Cured \\
\hline 13 & Male & 25 & Pul & $\mathrm{SS}+/ \mathrm{SC}+$ & $\begin{array}{l}\text { Pre-XDR }(F q): \\
\text { H, R, Z, E, Sm, } \\
\text { Ofx, Eto }\end{array}$ & 12 & $B / L(2) Y$ & $\begin{array}{c}\text { Lzd, Cfz, PAS, Cs, Cm, } \\
\operatorname{Mfx}(6)\end{array}$ & No & 4 & 3 & Cured \\
\hline 14 & Female & 18 & Pul & $\mathrm{SS}+/ \mathrm{SC}+$ & $\begin{array}{c}\text { XDR: } \\
\text { H, R, Z, E, Sm, } \\
\text { Ofx, Mfx, Eto, PAS, } \\
\text { Km, Am, Cm }\end{array}$ & 4 & $\mathrm{~B} / \mathrm{L}(3) ; \mathrm{Y}$ & $\begin{array}{c}\text { Lzd, Cfz, PAS, Cs, Clari, } \\
\text { Thr (6) }\end{array}$ & No & 5 & 4 & Cured \\
\hline 15 & Male & 29 & Pul & $\mathrm{SS}+/ \mathrm{SC}+$ & $\begin{array}{c}\text { XDR: } \\
\text { H, R, Z, E, Sm, } \\
\text { Ofx, Mfx, Eto, PAS, } \\
\text { Km, Am, Cm }\end{array}$ & 12 & $U / L(2) ; Y$ & $\begin{array}{c}\text { Lzd, Cfz, PAS, Cs, Cm, } \\
\operatorname{Mfx}(6)\end{array}$ & No & 6 & 2 & Cured \\
\hline 16 & Female & 29 & Pul & SS-/SC- & $\begin{array}{c}\text { XDR: } \\
\text { H, R, E, Sm, Ofx, } \\
\text { Mfx, Eto, PAS, Km, } \\
\text { Am, Cm }\end{array}$ & 7 & $B / L(3) ; Y$ & $\begin{array}{l}\text { Lzd, Cfz, PAS, Cs, Cm, } \\
\text { Eto, Mfx, Z (8) }\end{array}$ & No & 4 & $\begin{array}{l}\text { Negative at } \\
\text { baseline }\end{array}$ & Cured \\
\hline 17 & Female & 32 & Pul & $\mathrm{SS}-/ \mathrm{SC}+$ & $\begin{array}{l}\text { Pre-XDR (Fq): } \\
\text { H, R, Z, E, Sm, } \\
\text { Ofx, Mfx }\end{array}$ & 10 & $U / L(1) ; Y$ & $\begin{array}{c}\text { Lzd, Cfz, Cm, Eto, } \\
\text { Meropenem, Amox+Clav } \\
\text { (6) }\end{array}$ & No & 3 & 2 & $\begin{array}{l}\text { Sputum and culture } \\
\text { conversion (SS-/SC-, } \\
6 \text { months bedaquiline } \\
\text { completed) } \\
\text { OBR ongoing }\end{array}$ \\
\hline 18 & Female & 22 & Pul & $\mathrm{SS}+/ \mathrm{SC}+$ & $\begin{array}{c}\text { XDR: } \\
\text { H, R, Z, E, Sm, } \\
\text { Ofx, Eto, PAS, Km, } \\
\text { Am, Cm }\end{array}$ & 4 & $\mathrm{~B} / \mathrm{L}(3) ; \mathrm{Y}$ & $\begin{array}{l}\text { Lzd, Cfz, PAS, Cm, Lfx } \\
\text { (5) }\end{array}$ & $\begin{array}{l}\text { Yes (right upper } \\
\text { lobectomy) }\end{array}$ & 3 & 3 & $\begin{array}{c}\text { Sputum and culture } \\
\text { conversion (SS-/SC-, } \\
6 \text { months bedaquiline } \\
\text { completed) } \\
\text { OBR ongoing }\end{array}$ \\
\hline 19 & Female & 27 & Pul & $\mathrm{SS}+/ \mathrm{SC}+$ & $\begin{array}{c}\text { XDR: } \\
H, R, Z, S m, \text { Ofx, } \\
\text { Km, Am }\end{array}$ & 126 & $B / L(5) ; Y$ & $\begin{array}{l}\text { Lzd, Cfz, PAS, Cm, Eto, } \\
\text { Mfx, Amox+Clav, E (8) }\end{array}$ & No & 1 & - & $\begin{array}{c}\text { Nonresponder (SS+/SC+, at } \\
6 \text { months of bedaquiline) } \\
\text { Disease static } \\
\text { Currently SS+/SC+ } \\
\text { OBR ongoing }\end{array}$ \\
\hline 20 & Female & 29 & Pul & $\mathrm{SS}+/ \mathrm{SC}+$ & $\begin{array}{l}\text { Pre-XDR (Fq): } \\
\text { H, R, Z, E, Sm, } \\
\text { Ofx, Mfx, Eto }\end{array}$ & 74 & $U / L(3) ; Y$ & $\begin{array}{l}\text { Lzd, Cfz, PAS, Cs, Cm, } \\
\text { Lfx (6) }\end{array}$ & No & 2 & - & $\begin{array}{c}\text { Nonresponder (SS-/SC+, at } \\
6 \text { months of bedaquiline) } \\
\text { Disease static } \\
\text { Currently SS+/SC+ } \\
\text { OBR ongoing }\end{array}$ \\
\hline
\end{tabular}

OBR: optimised background regimen; XDR: extensively drug-resistant; SLD: second-line drug; Pul: pulmonary; Pl: pleural; SS: sputum smear; SC: sputum culture; H: isoniazid; R: rifampicin; Z: pyrazinamide; E: ethambutol; Sm: streptomycin; Eto: ethionamide; PAS: para-aminosalicylic acid; Fq: fluoroquinolone; Ofx: ofloxacin; Lfx: levofloxacin; Mfx: moxifloxacin; Km: kanamycin; Am: amikacin; Cm: capreomycin; Cs: cycloserine; Cfz: clofazimine; Lzd: linezolid; Amox: amoxicillin; Clav: clavulinic acid; Thr: thioridazine; Clari: clarithromycin; B/L: bilateral; U/L: unilateral; Y: yes for cavitation; N: no for cavitation; ${ }^{\#}:$ SLD duration prior to bedaquiline use; ${ }^{\Uparrow}$ : value in parentheses refers to the number of lobes affected; ${ }^{+}$: value in parentheses is the number of drugs; ${ }^{\S}$ : Time to culture conversion. 
All patients received $400 \mathrm{mg}$ of bedaquiline once per day for 2 weeks and then $200 \mathrm{mg}$ three times a week for a further 22 weeks in accordance with World Health Organization (WHO) guidelines [10] and in combination with an OBR of drugs chosen by the investigator based on individual DST reports. Linezolid and clofazimine were co-prescribed with bedaquiline in all our patients and, given their limited therapeutic options and documented susceptibility to clofazimine, all 20 patients received it prior to being initiated on bedaquiline. Cross resistance between clofazimine and bedaquiline was unlikely, as all six patients who failed to convert from a positive to a negative sputum culture despite receiving bedaquiline retained their susceptibility to clofazimine. In addition, although synergy between pyrazinamide and bedaquiline has been reported, the high incidence of resistance to pyrazinamide in our cohort (19 out of 20) resulted in it being omitted in these patients in order to reduce the pill burden and the adverse effects associated with it. No patient in this cohort received bedaquiline and delamanid at the same time during treatment. The mean number of drugs in the OBR was 5.6. Those who converted received a mean of six drugs while those who failed to convert received 4.7 drugs. Two patients underwent surgery just before receiving bedaquiline (table entries 11 and 12) while two underwent surgery after completing 24 weeks of bedaquiline (table entries 9 and 18). Three did well and were cured after 2 years of treatment while one remained culture positive (table entry 9 ).

All 20 patients completed 24 weeks of bedaquiline. Baseline TB sputum culture status was positive in 17 patients and negative in three. Of the 17 culture-positive patients, 11 converted at 6 months while six were persistently positive (table entries 5, 7-9, 19 and 20). The three patients who were sputum-culture negative before starting bedaquiline remained culture negative at 6 months. Thus, a total of 14 out of 20 patients were culture negative at the end of 6 months of bedaquiline while six remained positive. The time to culture conversion ranged from 1-5 months (mean 2.7 months); however, one patient (table entry 2) relapsed 16 months after original culture conversion. The average duration of prior SLD treatment was 22.9 months (8.53 months in those who culture converted and 49.57 months in the treatment failures). Cultures were more likely to convert in those patients where bedaquiline treatment was started relatively early. All 20 patients showed clinical improvement with a mean weight gain of $4.6 \mathrm{~kg}(6.1 \mathrm{~kg}$ among converters and $1.7 \mathrm{~kg}$ among treatment failures). Radiographic improvement occurred in 18 out of 20 patients.

The repurposed agents linezolid and clofazimine were co-prescribed with bedaquiline in all our patients and this might have contributed to the impressive $70 \%$ conversion rate, even in this heavily pre-treated patient cohort [11] with prolonged previous treatment and exposure to multiple SLDs. In addition to the duration of prior SLD use and the number of drugs in the OBR, acquired bedaquiline resistance [12] may have been a cause of treatment failure although we were unable to test for minimum inhibitory concentrations (MICs) for bedaquiline.

Of the six patients who were treatment failures, one (table entry 5) received delamanid for 24 weeks after her course of bedaquiline; however, her sputum continued to be positive for acid fast bacilli. Another patient (table entry 9) underwent a left pneumonectomy after completing the course of bedaquiline and is clinically stable, although repeat sputum cultures have been positive for Mycobacterium tuberculosis. Four other patients (table entries 7, 8, 19 and 20) are clinically stable. In addition, one patient (table entry 2) relapsed 16 months after culture conversion and bedaquiline, as well as delamanid, have been applied for. The OBR is being continued for all of the treatment failures.

All patients were carefully monitored but none suffered any major adverse effects. The QT-interval with Fridericia's correction (QTcF) increased by a mean of $49 \mathrm{~ms}$, which may be attributed to co-administration of bedaquiline and clofazimine in all 20 patients whereas a subset (five out of 20) also received moxifloxacin $[13,14]$. Three patients (table entries 2, 3 and 7) experienced a transient prolonging of the QTcF interval to above $500 \mathrm{~ms}$ in the region of 4-8 weeks from starting bedaquiline treatment (this reverted to below $500 \mathrm{~ms}$ by the next week). None of the patients were symptomatic and we found that bedaquiline and clofazimine were well tolerated together with neither drug requiring discontinuation.

Based on our experience, with excellent outcomes and acceptable safety being observed in this high-risk $\mathrm{CU}$ population, broader access to bedaquiline is needed since experience shows that it can be used successfully in Mumbai. Although the numbers are small, they seem to suggest better outcomes with earlier initiation of bedaquiline treatment. A conditional access programme for bedaquiline has been initiated with the donation of 600 patient courses to be used in six centres across the country. Presently, 64 patients have been enrolled for bedaquiline as part of this programme. Although none of our patients received concomitant treatment with bedaquiline and delamanid, clinical trials to study the feasibility of combining these drugs, as part of newer regimens for highly resistant TB patients who have limited treatment options, are urgently required [15].

Zarir F. Udwadia, Shashank Ganatra and Jai B. Mullerpattan

Dept of Respiratory Medicine, P.D. Hinduja National Hospital and Medical Research Center, Mumbai, India. 
Correspondence: Jai B. Mullerpattan, Dept of Respiratory Medicine, P.D. Hinduja National Hospital and Medical Research Center, Veer Savarkar Marg, Mahim, Mumbai, Maharashtra 400016, India. E-mail: jaimuller@hotmail.com

Received: Aug 022016 | Accepted after revision: Nov 222016

Conflict of interest: None declared.

\section{References}

1 Andries K, Verhasselt P, Guillemont J, et al. A diarylquinoline drug active on the ATP synthase of Mycobacterium tuberculosis. Science 2005; 307:223-227.

2 World Health Organization. Global tuberculosis report 2016. Geneva, World Health Organization, 2016.

3 Migliori GB, Sotgiu G, Gandhi NR, et al. Drug resistance beyond extensively drug-resistant tuberculosis: individual patient data meta-analysis. Eur Respir J 2013; 42: 169-117.

4 Dheda K, Gumbo T, Gandhi NR, et al. Global control of tuberculosis: from extensively drug-resistant to untreatable tuberculosis. Lancet Respir Med 2014; 2: 321-338.

5 Udwadia ZF, Amale RA, Ajbani KK, et al. Totally drug-resistant tuberculosis in India. Clin Infect Dis 2012; 54: 579-581.

6 Diacon AH, Pym A, Grobusch MP, et al. Multidrug resistant tuberculosis and culture conversion with bedaquiline. New Engl J Med 2014; 371: 723-732.

7 Guglielmetti L, Le Dû D, Jachym $\mathrm{M}$, et al. Compassionate use of bedaquiline for the treatment of multidrug-resistant and extensively drug-resistant tuberculosis: interim analysis of a French cohort. Clin Infect Dis 2015; 60: 188-194

8 Pym AS, Diacon AH, Tang SJ, et al. Bedaquiline in the treatment of multidrug- and extensively drug-resistant tuberculosis. Eur Resp J 2016; 47: 564-574.

9 Udwadia ZF, Moharil G. Multidrug-resistant-tuberculosis treatment in the Indian private sector: results from a tertiary referral private hospital in Mumbai. Lung India 2014; 31: 336.

10 World Health Organization. The use of bedaquiline to treat MDR-TB: interim policy guidance. Geneva, World Health Organization, 2013.

11 Sotgiu G, Pontali E, Migliori GB. Linezolid to treat MDR-/XDR-tuberculosis: available evidence and future scenarios. Eur Respir J 2015; 45: 25-29.

12 Bloemberg GV, Keller PM, Stucki D, et al. Acquired resistance to bedaquiline and delamanid in therapy for tuberculosis. New Engl J Med 2015; 373: 1986-1988.

13 Wallis R. Cardiac safety of extensively drug-resistant tuberculosis regimens including bedaquiline, delamanid and clofazimine. Eur Respir J 2016; 48: 1526-1527.

14 Tadolini M, Lingtsang RD, Tiberi $\mathrm{S}$, et al. Cardiac safety of extensively drug-resistant tuberculosis regimens including bedaquiline, delamanid and clofazimine. Eur Respir J 2016; 48: 1527-1529.

15 Tadolini M, Lingtsang RD, Tiberi S, et al. First case of extensively drug-resistant tuberculosis treated with both delamanid and bedaquiline. Eur Respir J 2016; 48: 935-938. 